Progress in Nuclear Science and Technology

Volume 6 (2019) pp. 43-47

\title{
ARTICLE
}

\section{Neutron and photon dose rate distributions in linac rooms}

\author{
Kazuaki Kosako ${ }^{\mathrm{a}^{*}}$, Kazuyoshi Masumoto ${ }^{\mathrm{b}}$, Koji Oishic ${ }^{\mathrm{c}}$, Hajime Nakamura ${ }^{\mathrm{b}}$, Akihiro Toyoda $^{\mathrm{b}}$, Kazuhiko Iijima ${ }^{\mathrm{b}}$ and $^{-}$ \\ Takashi Nakamura ${ }^{\mathrm{a}}$ \\ ${ }^{a}$ Shimizu Corporation, 3-4-17 Etchujima, Koto-ku, Tokyo, 135-8530, Japan; ${ }^{b}$ High Energy Accelerator Research Organization, 1-1 \\ Oho, Tsukuba-shi, Ibaraki-ken, 305-0801, Japan; ' Japan Environment Research Co. Ltd., 6-24-1 Nishi-shinjuku, Shinjuku-ku, \\ Tokyo, 160-0023, Japan
}

\begin{abstract}
In the X-ray irradiation of the $18 \mathrm{MeV}$ electron linac, the spatial distributions of neutron and photon ambient dose equivalent rates were measured by ADC plastic dosimeter and Optical Stimulated Luminescence dosimeter in the linac room. The spatial distributions were measured on the surface of the linac device, the irradiation room and the maze pathway. The calculations were executed for three types of linac head models with the MCNP5 code. The calculated accuracy of the spatial distribution was evaluated by comparing the measured results. The calculation-to-experiment ratios $(\mathrm{C} / \mathrm{E})$ of dose rates by the detailed linac head models converged to the range from 0.8 to 5 . The radioactivities of the concrete of linac room floor were measured and compared with the calculated result.
\end{abstract}

Keywords: dose rate; linac; bremsstrahlung; Monte Carlo; X-ray; radiotherapy; concrete radioactivity; spatial distribution; photoneutron

\section{Introduction}

Currently, the most cause of Japanese death is cancer and it occupies about $30 \%$ of the whole. As an effective treatment of cancer to keep a good quality of life, radiation therapy in Japan is estimated to have large increment. Electron linear accelerators (linacs) are used worldwide in radiation cancer therapy due to many clinical cases and cost benefit, and more than 900 linacs are being operated in Japan. Recently, the electron energy of greater than $10 \mathrm{MeV}$ (high-energy linac) is gradually increasing with the optimization of radiation cancer therapy and so on. When the electron energy is greater than $10 \mathrm{MeV}$, neutrons and charged particles are produced by photonuclear reactions through bremsstrahlung photons that are caused by electrons.

An analysis method to be used in patient dose estimation and shielding design for the high-energy linac is required to accurately estimate the behaviour of photons and photoneutrons. However, the verification of the transport calculation code including photoneutron reactions is insufficient for the inner space of electron linac facilities.

Here in this study, the neutron and photon ambient dose equivalent rates ("dose rates" for simplicity) and neutron fluxes in the linac room were measured using the $18 \mathrm{MeV}$ linac. The spatial distributions were measured on the surface of the linac device, the irradiation room and the maze pathway. The calculated

*Corresponding author. Email: k.kosako@shimz.co.jp accuracy of the spatial distributions of neutrons and photons with the Monte Carlo code MCNP5 [1] was evaluated by comparing the measured and calculated results. Furthermore, the radioactivity in the linac floor concrete was measured and the accuracy of calculated radioactivity was evaluated by the measured results.

\section{Experiment}

The experiment was performed at the first linac room of Hiroshima University Hospital, Hiroshima, Japan. The linac device was CLINAC 2300C/D manufactured by Varian Medical Systems, Inc. The copper target in the linac head was bombarded by $18 \mathrm{MeV}$ electrons and the maximum dose rate was $6 \mathrm{~Gy} / \mathrm{min}$ at IsoCenter position (IC). The irradiation direction was downward to floor in the experiment.

The spatial distributions of neutron and photon dose rates in the linac room were measured. The neutron dose rates were measured by ADC plastic dosimeter and the photon dose rates were made by Optical Stimulated Luminescence dosimeter (OSL) fabricated by Nagase-Landauer Co. Ltd. The neutron fluxes were measured by the activation detector of $\mathrm{Au}$ foils (20 $\mu$ m-thickness). The $\mathrm{Au}$ foils with or without the $\mathrm{Cd}$ cover ( $1 \mathrm{~mm}$-thickness) were made to obtain the thermal neutron fluxes.

The measured positions are classified into two types of locations on the wall surface in linac room and on the surface of linac device. Figure 1 shows the 14 positions 
of dosimeters measured in the linac room. The height of dosimeters in the room was $95 \mathrm{~cm}$ from the floor. Figure 2 shows the 11 positions of dosimeters measured around the linac head. The dosimeter position 22 is the reverse side of position 24 and the position 26 is the top of linac head.

To measure the radioactivities in the linac room, we obtained samples by core boring at four places from the linac floor concrete, as shown in Figure 3. No.2 core was used to component analysis and other three cores were used to measure the depth distributions of radioactivities.

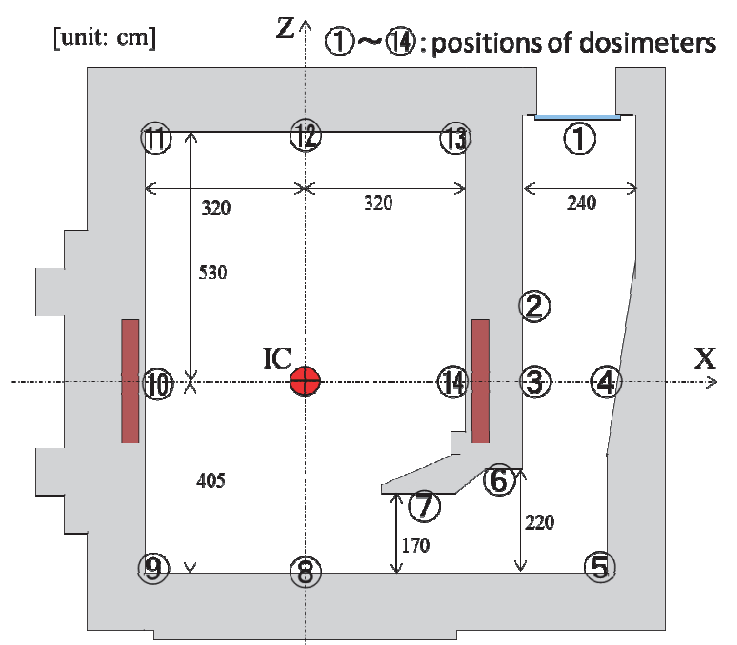

Figure 1. Layout of dosimeter positions in the linac room.

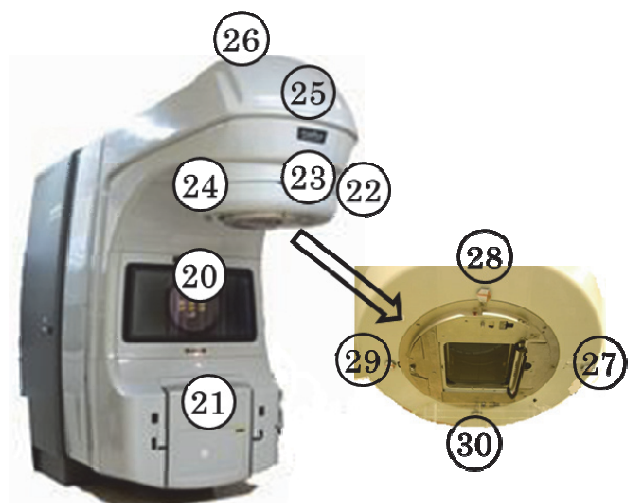

Figure 2. Layout of dosimeter positions around linac head.

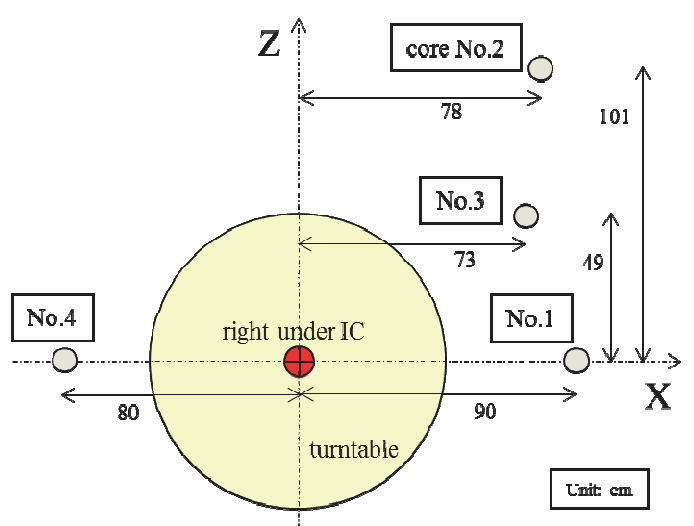

Figure 3. Layout of core boring positions of floor concrete.

\section{Calculation}

The photon and neutron fluxes at the measured positions in the linac room were calculated with the 3-dimensional Monte Carlo code MCNP5. The code was necessary to have functions of the electron-photon cascade and the photonuclear reaction, because the electron energy was greater than $10 \mathrm{MeV}$. The cross section data libraries for MCNP5 were used EL3 [2] for electron-photon cascade reactions, MCPLIB04 [2] for photon interaction, FSXLIB-J33 [3] or FSXLIB-J40 [4] for neutron reactions, and LA150U [2] for photonuclear reactions.
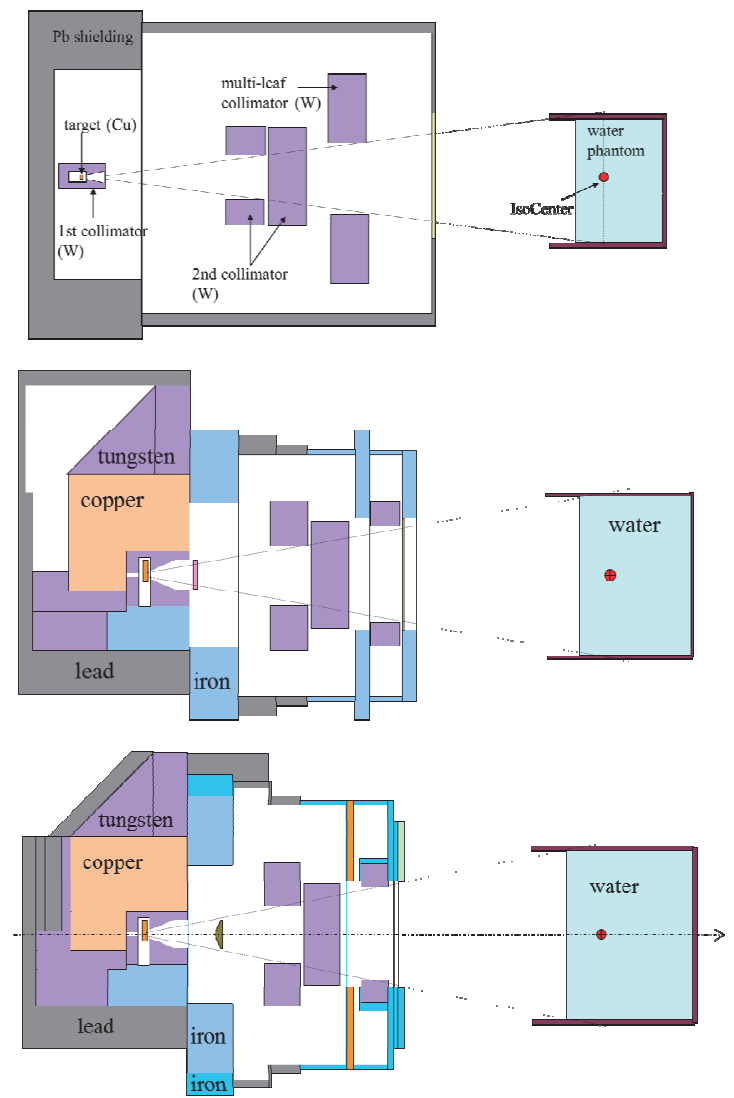

Figure 4. Schematic structures of the linac head models (simple, detailed II, and detailed III from top to bottom).

In the X-ray irradiation of the linac device, the electron beam was irradiated to back side of copper target and the bremsstrahlung photons from the front side of target were produced in the forward direction. The bremsstrahlung photons were formed by two tungsten collimators to have the maximum area of $40 \mathrm{x}$ $40 \mathrm{~cm}^{2}$ at IC. The target in the linac head module was surrounded with lead and tungsten shielding bodies and the photon leakage was largely limited to less than $1 / 1000$ with the shielding body. The linac head module had the complicated structure of the collimators, the flattening filter, the shielding body, the cables, and so on. Since the complete modelling of the head was very difficult, we made a simple model and 2 detailed head models to investigate the effect of modelling. The simple model consisted of the target, two collimators, 
multi-leaf collimator, and lead shielding cover. The detailed models (II and III) based on actual size consisted of the target, the flattening filter, two tungsten and multi-leaf collimators, the combined shielding bodies (tungsten, lead and iron), and the main part of linac, as shown in Figure 4. The colour of tungsten in Figure 4 is purple, lead is grey, iron is blue and light-blue, and copper is orange. The size of water phantom is $30 \times 30 \mathrm{~cm}^{2}$ and $20 \mathrm{~cm}$-depth and it is surrounded by the acrylic board of $1 \mathrm{~cm}$-thickness.

In the linac room shown in Figure 1, the irradiation area was $640 \mathrm{~cm}$-width, $935 \mathrm{~cm}$-depth, and 440 $\mathrm{cm}$-height. The widths of the foot-shaped maze gate were 170 and $220 \mathrm{~cm}$ and the maximum width of maze was $240 \mathrm{~cm}$. The density of the analyzed concrete was $2.23 \mathrm{~g} / \mathrm{cm}^{3}$. The flux-to-dose-rates conversion coefficients of neutron and photon were used [5].

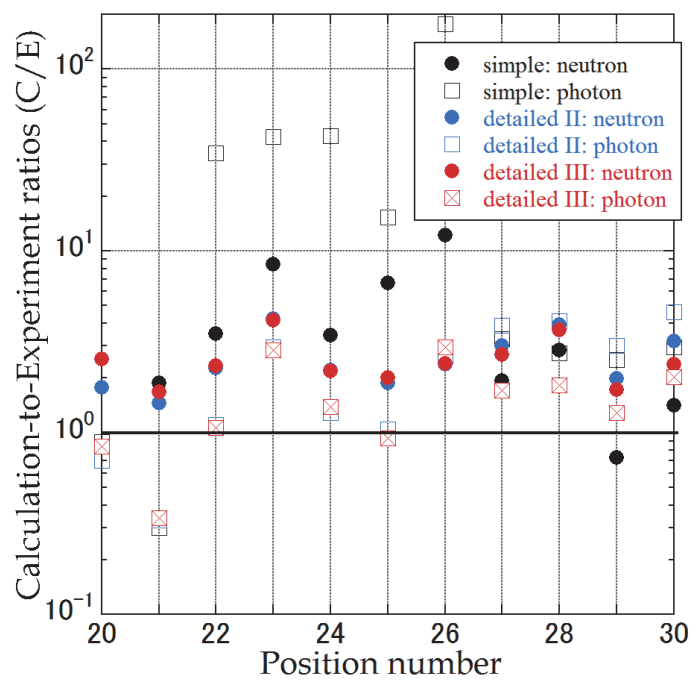

Figure 5. $\mathrm{C} / \mathrm{E}$ of neutron and photon dose rates around the 3 linac head models (in the case of downward irradiation of 18 MeV X-rays).

\section{Comparison between experiment and calculation}

\subsection{Dose rate distribution around the linac head}

At the measured positions around the linac head shown in Figure 2, neutron and photon dose rates were calculated for the simple and 2 detailed head models by MCNP5. Figure 5 shows the calculation-to-experiment ratios $(\mathrm{C} / \mathrm{E})$ of neutron and photon dose rates for 3 models. The experimental values were measured by $\mathrm{ADC}$ and OSL dosimeters. The linac operation condition was downward irradiation of $18 \mathrm{MeV}$ X-rays for one minute. In Figure 5, the black symbols show the simple head model, the blue show the detailed model II, and the red show the detailed model III. The filled circles show $\mathrm{C} / \mathrm{E}$ of neutron dose rates and the open squares show photon. Although the $\mathrm{C} / \mathrm{E}$ of the simple model spreads from 0.3 to 200 , the detailed models converge to the range from 1.5 to 5.0 for neutron and from 0.8 to 3.0 for photon, except for position 21 having strong position dependence to photons leaked from the complicated collimator system in the linac head. The leakage dose from the linac head can mainly calculate with overestimation of 2 to 3 times to measurement.

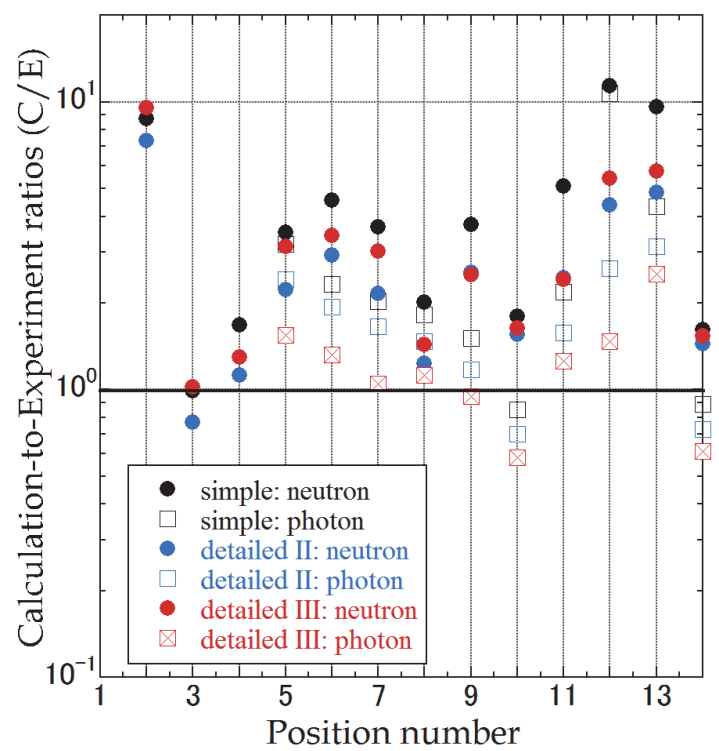

Figure 6. $\mathrm{C} / \mathrm{E}$ of neutron and photon dose rates by 3 head models in the linac room (in the case of downward irradiation of $18 \mathrm{MeV}$ X-rays).

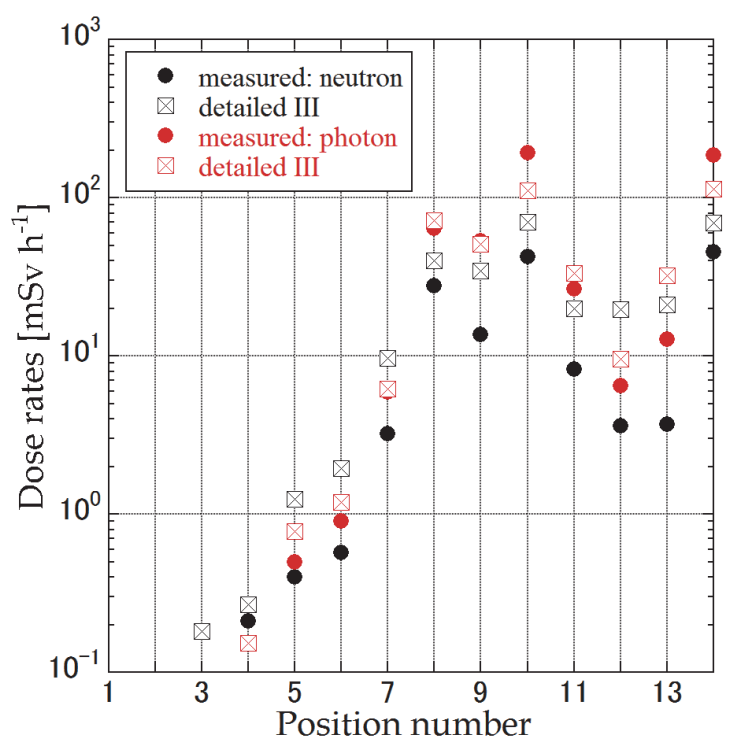

Figure 7. Dose rates of neutron and photon by the measurement and the detailed model III in the linac room.

\subsection{Dose rate distributions in the linac room}

At the measured positions on the wall surface in the linac room shown in Figure 1, neutron and photon dose rates were calculated for 3 models. Figure 6 shows the $\mathrm{C} / \mathrm{E}$ of neutron and photon dose rates in the linac room. The range of $\mathrm{C} / \mathrm{E}$ of the simple model is from 0.9 to 11 and the detailed models are from 0.8 to 3.5 for neutron, except for positions 1, 12 and 13, and from 0.6 to 3.0 for photon. The simple model overestimates of 1.5 to 2 times to the detailed model. The positions from 11 to 13 are affected by the main body of linac. Figure 7 shows 
the neutron and photon dose rates in the linac room. The positions 1 and 2 are lower than the detection limit or $0.1 \mathrm{mSv} / \mathrm{h}$. The trend of dose rate distribution gives good agreement between the measurement and the calculation with detailed model III.

\subsection{Neutron flux distributions in the linac room}

At the measured positions shown in Figure 1, the thermal neutron and epi-thermal neutron fluxes were measured by the $\mathrm{Au}$ foils with or without the $\mathrm{Cd}$ cover. Figure 8 shows the $\mathrm{C} / \mathrm{E}$ of thermal and epi-thermal neutron fluxes in the linac room. In this calculation, the linac head uses the detailed head model III, the energy range of thermal neutron is less than $0.876 \mathrm{eV}$ by the $\mathrm{Cd}$ cover and the epi-thermal neutron is from 0.876 to 10.7 $\mathrm{eV}$. The range of $\mathrm{C} / \mathrm{E}$ of thermal neutron flux is mainly from 1.0 to 2.0 and the calculated result is good agreement with the measurement. The range of $\mathrm{C} / \mathrm{E}$ of epi-thermal neutron flux is from 2 to 11 and the calculated result is large overestimation. The thermal neutron is more important than the epi-thermal neutron, because the thermal neutron flux is about 10 times of the epi-thermal.

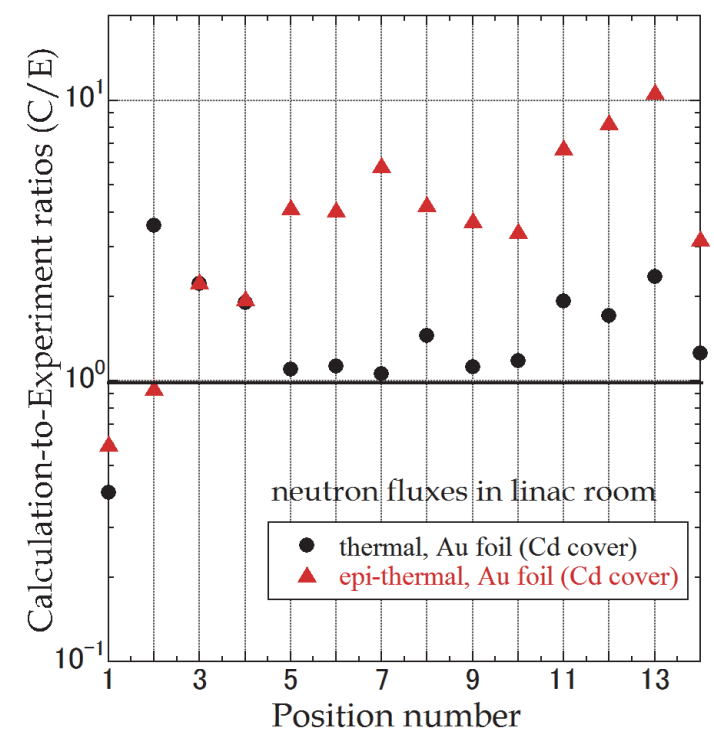

Figure 8. $\mathrm{C} / \mathrm{E}$ of thermal and epi-thermal neutron fluxes in the linac room to the measurement by Au foil.

\subsection{Radioactivity of the linac floor concrete}

At the core boring positions of the linac floor concrete shown in Figure 3, the radioactivities of ${ }^{60} \mathrm{Co}$ and ${ }^{152} \mathrm{Eu}$ were calculated by the DCHAIN-SP2001 code [6] based on the $18 \mathrm{MeV}$ irradiation record of the linac operation period for 15 years. The calculations were performed by the continuous irradiation divided in first 10 years and later 5 years. The neutron energy spectra of concrete core used in the radioactivity calculation were obtained by an interval of $2 \mathrm{~cm}$-depth from the MCNP5 calculation, as shown in Figure 9. The concrete composition based on components analysis of core No. 2 was used to the radioactivity calculation. The mass spectrometry of $\mathrm{Co}, \mathrm{Cs}$ and $\mathrm{Eu}$ which were important impurity elements used the ICP-MS method. Figure 10 shows the radioactivities of ${ }^{60} \mathrm{Co}$ and ${ }^{152} \mathrm{Eu}$ measured the core No.1, 3 and 4 and calculated the core No.4. For ${ }^{60} \mathrm{Co}$ in the core No.4, the agreement between the measurement and calculation is good. But the calculated values of ${ }^{152} \mathrm{Eu}$ give $50 \%$ underestimation to the measured data. In the comparison among core No.1, 3 and 4 , the difference between calculations is less than $20 \%$ and the difference between measurements is from $50 \%$ to $120 \%$. The variation of measured data is large than it of neutron fluxes. Thus the abundance of Co and Eu which are minor impurities in concrete has strong localization.

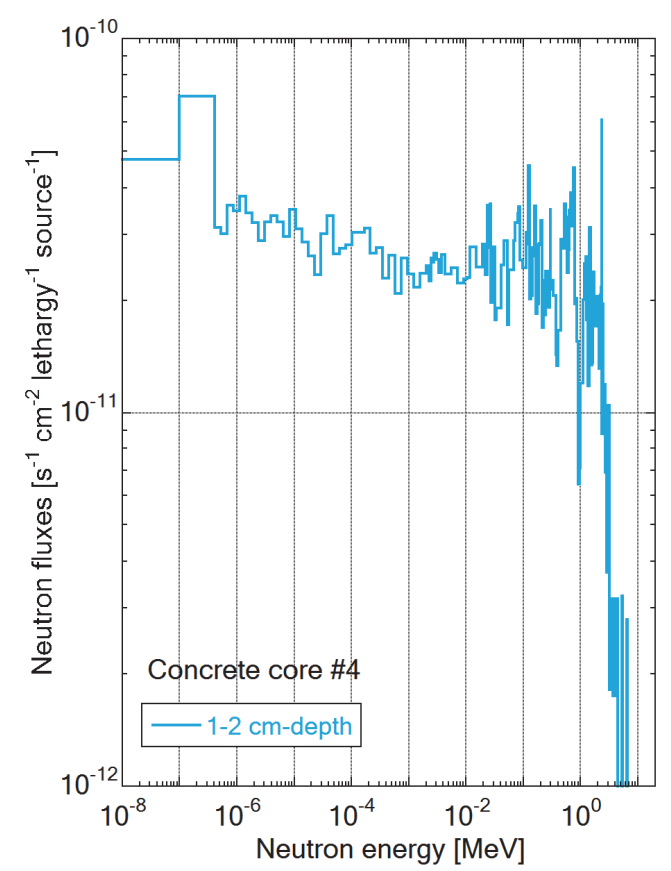

Figure 9. Neutron energy spectrum at 1 to $2 \mathrm{~cm}$-depth in core No. 4 of the linac room floor concrete.

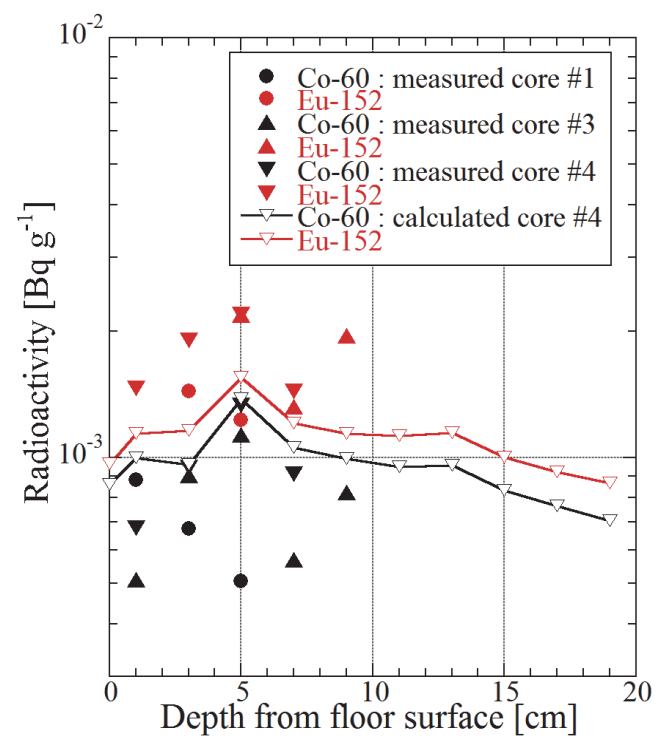

Figure 10. The radioactivities of ${ }^{60} \mathrm{Co}$ and ${ }^{152} \mathrm{Eu}$ in core No. 1, 3 and 4 of the linac room floor concrete. 


\section{Conclusion}

We measured the spatial distributions of neutron and photon in the $18 \mathrm{MeV}$ electron linac room by 2 kinds of dosimeters and Au foils. The measured positions were locations around the linac head and on the wall surface in the linac room. The MCNP5 calculations were executed to the simple model and the two detailed models of linac head. The detailed model was necessary to estimate the neutron and photon dose rate distributions. The $\mathrm{C} / \mathrm{E}$ of detailed model was from 0.8 to 5 around the linac head and was from 0.6 to 3.5 in the linac room.

We measured the radioactivities in the cores of the linac floor concrete. The calculated results of ${ }^{60} \mathrm{Co}$ and ${ }^{152} \mathrm{Eu}$ agree with the measured data within $50 \%$ accuracy.

\section{References}

[1] X-5 Monte Carlo Team, MCNP - A General Monte Carlo N-Particle Transport Code, Version 5, LA-CP-03-0245, Los Alamos National Laboratory,
(2003).

[2] RSICC, MCNP6.1/MCNP5/MCNPX, CCC-810, Oak Ridge National Laboratory, (2013).

[3] K. Kosako, N. Yamano, T. Fukahori, K. Shibata and A. Hasegawa, The libraries FSXLIB and MATXSLIB based on JENDL-3.3, JAERI-Data/Code 2003-011, Japan Atomic Energy Research Institute, (2003).

[4] RIST/Code-Center, FSXLIB-J40: Neutron Cross Section Library in ACE Format for Continuous Energy Monte Carlo Codes, Research Organization for Information Science and Technology, (2010).

[5] Standards Committee of AESJ, Radiation dose conversion coefficients for radiation shielding calculations: 2010, AESJ-SC-R002:2010, Atomic Energy Society of Japan, (2010).

[6] T. Kai, F. Maekawa, K. Kosako, Y. Kasugai, H. Takada, and Y. Ikeda, DCHAIN-SP 2001: high energy particle induced radioactivity calculation code, JAERI-Data/Code 2001-016, Japan Atomic Energy Research Institute, (2001). 